\title{
El primero de la clase (2008): un reto de aceptación social
}

\author{
Victoria NOVELO-ALCOCER, Reinhard JANSSEN-AGUILAR, Mario Alberto LIZAMA-RUBIO, Nina Isabel \\ MÉNDEZ-DOMÍNGUEZ
}

Universidad Marista de Mérida, Campus de Ciencias de la Salud, Mérida, Yucatán (México).

Autor para correspondencia: Nina Isabel Méndez Domínguez. Correo electrónico: ninuxka@hotmail.com

Recibido el 22 de octubre de 2018; aceptado el 4 de diciembre de 2018.

Cómo citar este artículo: Novelo Alcocer V, Janssen Aguilar R, Lizama Rubio MA, Méndez Domínguez NI. El primero de la clase (2008): un reto de aceptación social. Rev Med Cine [Internet] 2019;15(2): 95-100.

DOI: http://dx.doi.org/10.14201/rmc201915295100

\section{Resumen}

La película El primero de la clase (2008) de Peter Werner, se encuentra basada en la vida de Brad Cohen, quien a partir de los 6 años empiezó a presentar manifestaciones clínicas del síndrome de Tourette. La trama se desarrolla entorno a como Brad vive su día a día con su enfermedad y cómo este logra enfrentar los diversos obstáculos que la sociedad le presenta para lograr su más grande sueño: ser profesor de escuela primaria. Brad es continuamente burlado por parte de sus compañeros, convive con profesores intolerantes e incluso tiene un padre muy poco comprensivo, esto debido a que se desconocía que era un portador de Síndrome de Tourette (ST), lo cual generó que en un principio se aislara de los demás y no quisiera interactuar con otras personas. Durante el filme observamos como continuamente Brad es víctima del estigma social al no poder comportarse como los demás a causa del ST, impidiéndole en un principio tener amigos y posteriormente conseguir trabajo. En el presente artículo, ofreceremos una revisión del padecimiento, para comprender sus manifestaciones y el modo en el que afectaron la vida de Brad.

Palabras clave: síndrome de Tourette, signos y síntomas, enfermedad del sistema nervioso.

\section{Front of the Class (2008): A challenge of Social Acceptance}

\section{Summary}

The film Front of the class (2008) by Peter Werner, is based on the life of Brad Cohen, from the age of 6 presented clinical manifestations of Tourette syndrome. The plot describes how Brad lived his day to day with his illness and how he managed with the various obstacles that society presented to achieve his greatest dream: to be an elementary school teacher. Brad was continuously bullied by his peers, he had intolerant teachers and even a very unsympathetic father, perhaps because his father did not know that he was a carrier of Tourette syndrome (TS), a disease that involves social isolation from the others and aversion to interact with other people. During the film we observe how Brad is a victim of social stigma by not being able to behave like this peers because of the TS, preventing him in the beginning to have friends and then and then, to get a job. In this article, we will offer a review of the Tourette's Syndrome to understand its manifestations and the way in which Brad's life was affected.

Keywords: Tourette Syndrome, Signs and Symptoms, Nervous System Disease. 


\section{Ficha técnica}

Título: El primero de la clase (TV). Título original: Front of the class (TV), aka Hallmark Hall of Fame: Front of the Class (TV).

País: Estados Unidos.

Año: 2008.

Director: Peter Werner.

Música: Ernest Troost.

Fotografía: Paul Elliot.

Montaje: David Beatty.

Guión: Thomas Rickman sobre el libro biográfico Front of the Class de Brad Cohen y Lisa Wysocky.

Intérpretes: Jimmy Wolk, Treat Williams, Dominic Scott Kay, Sarah Drew, Kathleen York, Joe Chrest, Patricia Heaton, Johnny Pacar, Charles Henry Wyson, Charlie Finn.

Color: color.

Duración: 95 minutos.

Género: drama, discapacidad, educación, enfermedad, película de televisión.

Sinopsis: "Siendo retado desde muy joven por el síndrome de Tourette, Brad Cohen desafía todas las posibilidades para convertirse en un maestro talentoso. A medida que crece, él debe enfrentarse a amigos y compañeros de clase quienes no se dan cuenta que a veces no puede controlar sus ataques repentinos, y a un padre que al parecer no quiere aceptar la condición de su hijo" (IMDb).

Productoras: Hallmark Hall of Fame Productions, McGee Street Productions.

\section{Enlaces:}

https://www.imdb.com/title/tt1292594 https://www.filmaffinity.com/es/film725838.html

\section{Tráiler en inglés}

\section{Introducción}

Brad, portador del Síndrome de Tourette, narra en El primero de la clase desde su propia voz, cómo logró alcanzar su sueño de ser maestro de escuela primaria, afrontando todos los obstáculos que la sociedad le fue planteando.

Brad se refiere a su enfermedad como a un "amiguito" que se manifiesta desde que él tiene 6 años e inmediatamente, desde ese momento, empieza a afectar su vida personal en el ámbito familiar y social, impidiéndole

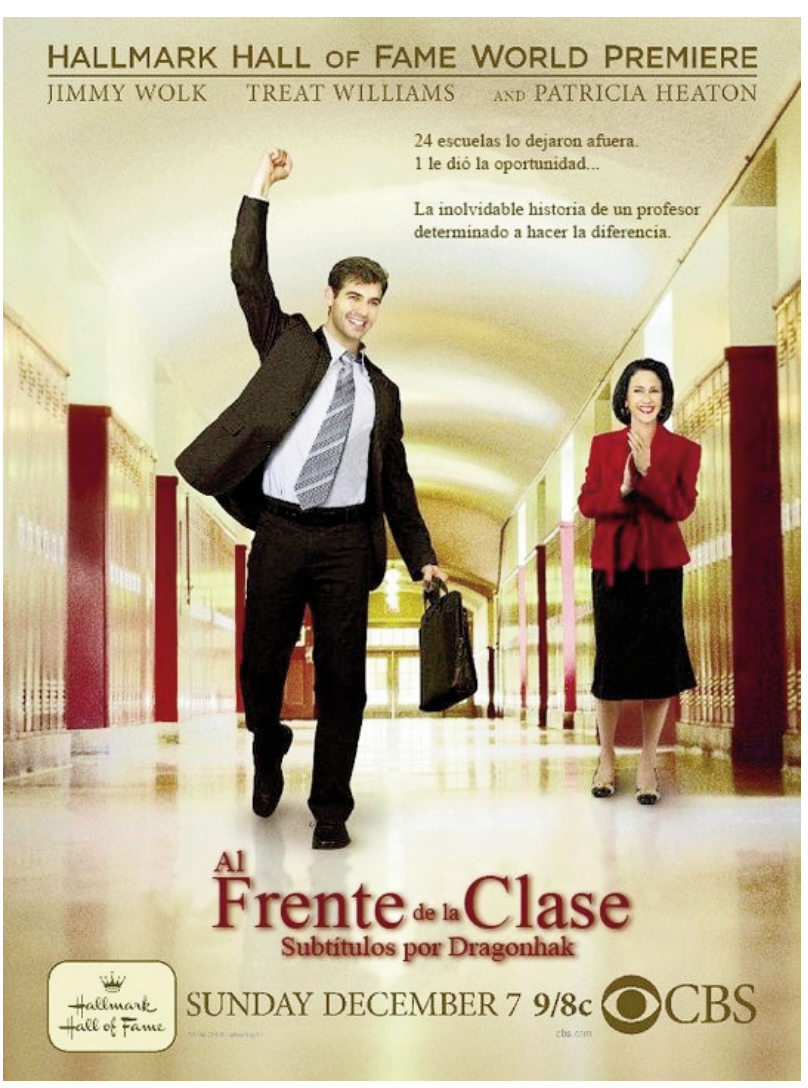

establecer relaciones interpersonales sólidas con sus padres, compañeros de escuela y profesores.

Los tics que el ST le producía, eran mal vistos por los demás que lo rodeaban, generando estigma y conllevando a que constantemente fuera reprendido y castigado por los mismos, ya que eran considerados como un intento de burla, a tal grado que su propio padre llegó a creer que los tics tenían la intención retarlo y restarle autoridad. Sin embargo, su madre constantemente preocupada por él, buscó asesoría médica, pero incluso en ese gremio, los profesionales creían que quería llamar la atención.

El matrimonio de los padres de Brad se vio deteriorado y terminaron por divorciarse. La madre de Brad nunca se rindió, siendo clave en el correcto diagnóstico de la enfermedad de su hijo. Por su cuenta investigó en libros de medicina, y en uno de ellos, encontró información sobre el síndrome de Tourette, la cual corroboró con un psiquiatra. A partir de ese momento, Brad comenzó un camino hacia la comprensión de su padecimiento. Superando cualquier expectativa, Brad logró terminar sus estudios universitarios para poder enseñar como profesor en primaria, pero a pesar de ello, tuvo que afrontar un nuevo reto: conseguir trabajo en una escuela. 
Constantemente acudía a entrevistas en diferentes escuelas, pero su "amiguito" acudía en medio de las mismas, generando duda sobre las capacidades de Brad para ser profesor y propiciando que este no fuera aceptado. Esto fue generando que en Brad surgiera un sentimiento de impotencia ante el no poder controlar la enfermedad, generando ansiedad y temor que al final logró por superar gracias a su familia y amigos. Su madrastra le hizo comprender que no es la enfermedad quien lo está limitando, sino es el mismo quien se está limitando. Al escuchar estas palabras Brad se dio cuenta que el salir adelante está en sus manos, y como dice al final: "El mejor maestro que he tenido en la vida ha sido el Síndrome de Tourette".

Pero ¿cómo es que este padecimiento afecta la socialización y las relaciones interpersonales y por qué no fue sospechado más tempranamente dadas las manifestaciones clínicas de Brad?, en el presente artículo, ofreceremos una revisión del padecimiento para comprender sus manifestaciones y el modo en el que afectaron la vida de Brad.

\section{Síndrome de Tourette}

Este síndrome recibió su nombre del médico Georges Albert Édouard Brutus Gilles de la Tourette (1857-1904), un neurólogo francés quien en 1885 describió por primera vez la afección de manera conjunta con su mentor, el doctor Jean Martin Charcot. Juntos estudiaron diferentes pacientes con el objetivo de redefinir todos los trastornos del movimiento que, hasta entonces, se consideraban alteraciones incluidas en la corea o en la histeria ${ }^{1}$.

En 1980, en el DSM-III, el trastorno de Tourette (TT) fue incluido dentro de los trastornos de inicio en la infancia, la niñez o la adolescencia en el apartado de trastornos del movimiento estereotipado, donde se incluían el trastorno de tics transitorio, el trastorno de tics motores crónicos, el trastorno de Tourette, el trastorno de tics atípico y el trastorno del movimiento estereotipado atípico².

EI TT es el resultado de una disfunción cerebral frontoestriatal que afecta a personas de todas las edades; es un trastorno neurológico que se caracteriza por la aparición de tics motores y fónicos de manera precoz y fluctuantes típicamente que se notan por primera vez en la infancia, con inicio promedio entre los 3 y 9 años de edad, con continuación en la adolescencia y la adultez; aparece en personas de todos los grupos étnicos, es más común en hombres que en mujeres a razón de 4:11,3.
Los tics se explican por cambios en el funcionamiento frontoestriatal, al detectarse variaciones estructurales en los ganglios basales, como la disminución del volumen del núcleo caudado, el putamen y el globo pálido, especialmente en el hemisferio izquierdo, así como un hipometabolismo estriatal ${ }^{3}$.

Los niños con TT se caracterizan por la presencia de tics, esto se refiere a movimientos involuntarios, repetitivos y estereotipados de tipo motor y/o fónico, precedidos por sensaciones premonitorias descritas como sensación de hormigueo o picor en la zona donde posteriormente aparece el tic ${ }^{4}$.

Existen dos clasificaciones según la sintomatología del grupo muscular implicado:

- Motores: involucran a músculos esqueléticos, que a su vez pueden ser:

a. Simples: un solo músculo o un grupo muscular (por ejemplo: cierre palpebral, giros cervicales, elevación del hombro.) y

b. Complejos: que provocan un movimiento más elaborado y afectan a varios grupos musculares.

- Vocales o fónicos: afectan a músculos faringolaríngeos, y se diferencian en:

a. Simples: vocalizaciones o ruidos simples, y

b. Complejos: que se expresan como lenguaje articulado $^{4}$. Incluyen coprolalia (balbucear palabras socialmente inapropiadas tales como decir palabrotas) o ecolalia (repetir las palabras o frases de los demás), sin embargo, la coprolalia solamente está presente en un pequeño número (10 a 15 por ciento) de individuos con TT. A menudo, los tics empeoran con la excitación o la ansiedad y mejoran durante actividades en calma y enfocadas ${ }^{1}$.

El diagnóstico sigue siendo fundamentalmente clínico, basado en los criterios del DSM-IV-TR. Se suelen realizar estudios de neuroimagen y/o analíticos para descartar las causas de tics secundarios 4 .

Una buena parte de los casos de ST tienen un componte hereditario. Los estudios familiares tempranos sugieren un modo autosómico dominante de herencia (en el cual una sola copia del gen defectuoso heredado de uno de los padres es necesaria para producir el trastorno) ${ }^{1}$. Ocasionalmente se encuentran mutaciones de NOVO y se han identificado cuatro genes en los cuales se han producido mutaciones en diferentes pacientes de modo independiente: WWC1, CELSR3, NIPBL y FN1.

La presencia de mutaciones en estos genes aumenta significativamente el riesgo a desarrollar la enfermedad. WWC1 codifica para una proteína del citoplasma que se expresa en riñón y cerebro e interacciona 
con múltiples rutas moleculares. CELSR3 codifica para una proteína cadherina que podría estar relacionada con el crecimiento de las neuritas y la función de los axones en neuronas. NIPBL codifica para una proteína relacionada con la reparación del ADN y la unión de cromátidas durante la meiosis. Por último, FN1 codifica para dos formas de la proteína fibronectina 1 implicadas en la adhesión celular, la migración y la diferenciación celular 5 .

Un 90\% de los casos de ST se presentan con comorbilidades tales como: ansiedad, depresión, trastornos de aprendizaje y del sueño, sin embargo, los más comunes son: el trastorno por déficit de atención e hiperactividad (TDAH) y el trastorno obsesivo compulsivo (TOC) ${ }^{6}$.

El tratamiento médico del ST va enfocado a las comorbilidades, lo cual contribuye a disminuir la frecuencia e intensidad de los Tics. Por otra parte, dentro del tratamiento, se encuentra el ámbito psicológico, lo cual incluye la Intervención conductual integral (ICI) y la terapia de aceptación y de compromiso. El estándar de oro del tratamiento es la ICI la cual busca que el paciente con ST esté al tanto de las urgencias premonitorias de los tics y este pueda actuar de cierta manera respondiendo de una manera adecuada 7,8 .

\section{Síndrome de Tourette y comorbilidades}

La depresión y la ansiedad juegan un papel muy importante en las personas con ST debido a que, como mencionan diferentes estudios, estas comorbilidades se consideran factores de riesgo para el aumento de la sintomatología, es decir, el inicio y la exageración de los movimientos involuntarios, siendo la mayoría de las veces el factor mas importante por el cual la persona se rehúsa a realizar las actividades cotidianas de la vida e incluso a realizar actividades recreativas públicas en las cuales puedan ser juzgados por la ignorancia de la gente hacia la enfermedad 8 .

La coexistencia del ST, más el trastorno de déficit de atención e hiperactividad, como se mostró en el caso de Brad, genera que los pacientes se aíslen de la sociedad por miedo a ser rechazados y a ser juzgados, esto lleva consigo a que las personas con este trastorno puedan incluso llegar al estado de depresión mayor en la cual existe mayor ansiedad y estrés con el siguiente aumento de los periodos sintomáticos incontrolables y deteriorando su calidad de vida.

Los estudios cualitativos han revelado que el impacto negativo en la poca calidad de vida de los pacientes con ST proviene principalmente del deseo de los jóvenes de poder controlar los TICS, la expectativa de la sociedad de un comportamiento normal y la angustia generada por las urgencias premonitorias de los ataques $^{9}$. En la película podemos apreciar como en las diferentes etapas de su vida Brad Cohen se topa con estas tres situaciones antes mencionadas. Un ejemplo muy claro es cuando él se encuentra en clase en la escuela primaria, se puede apreciar que cuando siente la urgencia de realizar los tics se coloca un lápiz en la boca para poder controlarlos (Foto 1). Las personas con tics normalmente los pueden contener temporalmente, sin embargo, una vez que estos se producen atraen la atención de otras personas y esto lleva a problemas en las relaciones interpersonales ${ }^{10}$. En la escena antes mencionada Brad no logra controlar la urgencia de los tics, y la profesora tacha al pequeño Brad como niño problema, incluso al grado de hacerlo disculparse con sus compañeros por interrumpir a "propósito" el estudio de ellos, generando una relación conflictiva con sus compañeros.

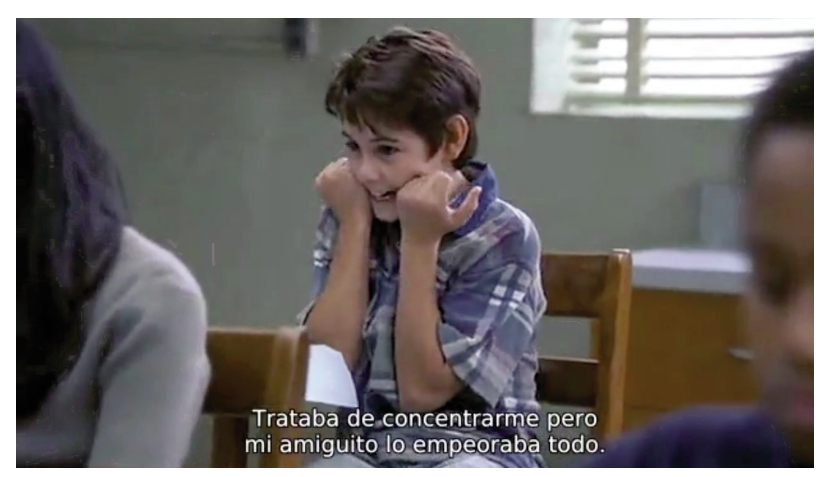

Foto 1. Brad Cohen se coloca un lápiz en la boca durante una clase para intentar controlar los tics.

\section{Socialización en los pacientes con Síndrome de Tourette}

En las relaciones interpersonales, el lenguaje simbólico se interpreta como parte de la comunicación. Los individuos no pueden adoptar ninguna acción hasta que ellos entiendan el contexto social de la acción, lo que les permite determinar cuál es una conducta apropiada en dichas circunstancias ${ }^{11}$. La autoconformación de los individuos se da través de su autopercepción, otros significativos y grupos de referencia ${ }^{12}$. Se ha visto que los adolescentes con ST, en comparación con sus compañeros sanos, evitan tener relaciones interpersonales, tienen diversos problemas emocionales y de comportamiento, son retraídos y en consecuencia se vuelven impopulares y silentes (Foto 2). Esto como consecuencia de los tics ${ }^{10}$. Durante la película, Brad es víctima del estigma que la sociedad genera ante su condición. Al inicio, mientras 


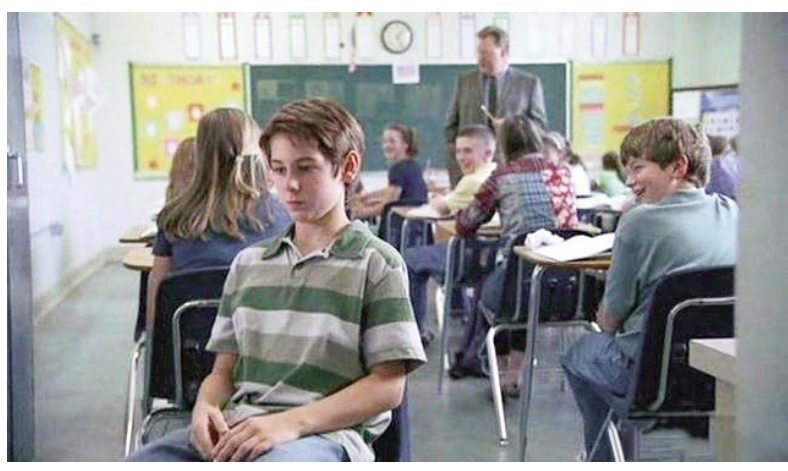

Foto 2. Brad es constantemente aislado de los demás por efecto de sus tics.

comienza a narrar parte de su historia, podemos ver cómo mientras maneja una patrulla de policía lo detiene por ir por arriba del límite de velocidad. Mientras Brad es interrogado se le presenta un ataque de tics y el policía le pregunta si Brad había estado bebiendo.

Los padres con hijos portadores del síndrome de Tourette reportan niveles elevados de estrés familiar comparado con padres con hijos no portadores. Estos reportes reflejan que el estrés familiar va a ser reflejado en el comportamiento del niño afectado creando niveles elevados de ansiedad, depresión y estrés ${ }^{13}$. Un punto importante a dejar en claro, es que la ruptura familiar solo empeora el trastorno al orientar al niño a la falta de apoyo familiar, a crear un personalidad anti social y de rechazo y al aumento de comorbilidades como la depresión ${ }^{14}$.

Los adolescentes con ST normalmente se encuentran preocupados porque sus compañeros sin ST no comprendan que sus ataques de tics son involuntarios. Estos mismos batallan para integrarse a la sociedad y por lo tanto, intentan desesperadamente esconder sus síntomas y tratan de vivir imitando según lo que la sociedad plantea como adecuado ${ }^{15}$.

Estas creencias negativas sobre los tics generan incomodidad. Es por esto que las personas con ST erróneamente creen que el sentir incomodidad se traduce como una situación catastrófica, intolerable y de larga duración y que vivir con esta incomodidad es muy difícill$^{8}$. En la escena de la película, donde Brad es llevado de nueva cuenta con el director por interrumpir con sus tics la clase de matemáticas, este lo invita a asistir a una presentación de la orquesta escolar, a lo que Brad responde que no, ya que no quiere causar "problemas". En otra escena, ya con el Brad adulto y siendo profesor, cuando asiste al funeral de uno de sus alumnos, se puede ver como él no quiere entrar, por temor a perjudicar con sus tics el funeral. En estas dos situaciones se puede ver como el personaje principal percibe los tics como algo negativo, perjudicial y que el hecho de que se presentaran, pudiera terminar en su desaprobación por la sociedad. De igual forma estas sensaciones negativas se pueden apreciar en cada una de sus entrevistas, que le generaban problemas para ser tomado en serio y que al final, en su última entrevista, llegó al punto de la desesperación e ira por la impotencia de no poder controlar sus tics (Foto 3).

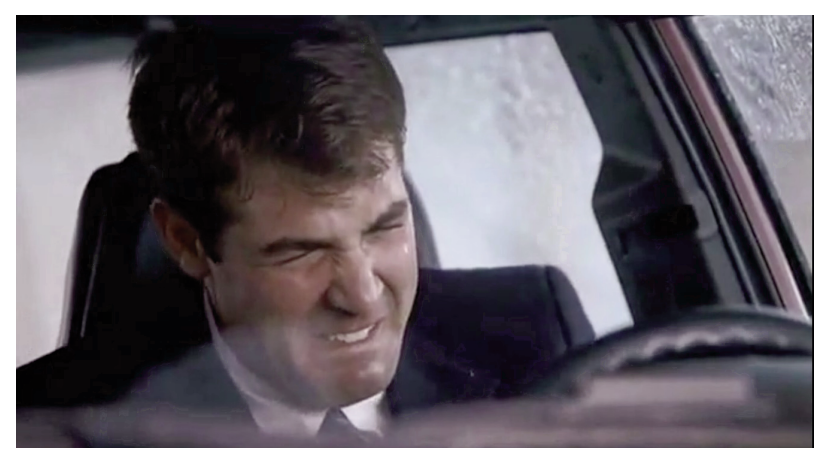

Foto 3. Dan saliendo de su entrevista número 24 en dónde pusieron en cuestión sus capacidades por culpa del ST. Esto le genera impotencia e ira.

En un estudio se vio que para que los adolescentes con ST se identifiquen a ellos mismos y puedan coexistir con su enfermedad, fue necesario que recibieran apoyo de sus profesores en la escuela y que reciban reconocimiento de sus pares. Esto a través de que se concientizara a los profesores y estos a los alumnos sin ST. Esto permitió que los adolescentes sin ST establecieran relaciones sólidas con sus pares, aumentando su autoestima y llegando a afirmar que la alegría de ir a la escuela les permitía olvidarse temporalmente de sus tics ${ }^{10}$. Un ejemplo de lo anterior fue cómo el director que invitó a Brad a la presentación de la orquesta de su escuela, al final de la misma, le pidió a Brad que les explicara a todos en qué consistía su enfermedad (Foto 4), y a partir de eso, la situación del joven Brad mejoró, demostrando que concientizando a los demás sobre la enfermedad mejora la calidad de vida de los afectados.

Otra parte importante es la aceptación de uno mismo con la enfermedad, a identificar las urgencias premonitorias y a aprender a actuar en base a ellas. Esto a través de la $\mathrm{ICl}$ y las terapias de aceptación y compromiso, logrando una disminución en la presentación de los tics. En la película Brad no recibe terapia, sin embargo, con ayuda de su madrastra, luego de su última entrevista que resultó ser un desastre, ésta le dice que no es su enfermedad la que lo detiene, sino es él quien se está frenando. Brad ante esto se da cuenta que debe coexistir con 


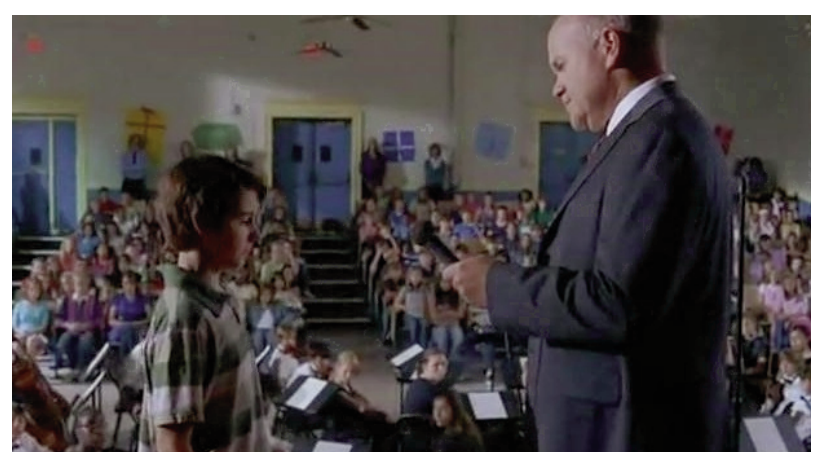

Foto 4. El director de la escuela le pide a Brad que le explique a los demás sobre su enfermedad.

sus tics y aceptarlos como son y demostrar que no son un impedimento para que pueda ejercer como profesor 7,8 .

\section{Conclusión}

La sociedad crea patrones de conducta a seguir, de lo que es un comportamiento adecuado y de lo que no se considera como tal. Salirse o desviarse de estos patrones de comportamiento, como los que genera el síndrome de Tourette, aunado al desconocimiento de esta enfermedad, genera estigma social conllevando a la discriminación y aislamiento de estas personas, perjudicando su calidad de vida. Esto pudiera ser evitado con una correcta concientización de la población y actualización de las normas sociales que sean más inclusivas y tolerantes.

\section{Referencias}

1. National Institutes of Health. Síndrome de Tourette. Washington D. C.: U.S. Department of Health and Human Services; 2012.

2. Carmona Fernández C, Artigas Pallarés J. El trastorno de Tourette a lo largo de la historia. Rev Asoc Esp Neuropsiq. 2016;36(130):347-62.

3. Espert R, Gadea M, Aliño M, Oltra-Cucarella J. Neuropsicología del trastorno de Tourette: cognición, neuroimagen y creatividad. Rev Neurol. 2017;64(10):S1-S8. 4. Muñoz Farjas E, Garcés Redondo M, Baiges Octavio J.J. Tratamiento de los tics y trastornos asociados. Rev Esp Trastor Mov. 2009;2(3):6-15.

5. Weiler N. First Clear-Cut Risk Genes for Tourette Disorder Revealed, Results Point to 'Bright Future' for Understanding Disorder. University of California San Francisco. News \& Media [Internet]. 3 de mayo de 2017. [citado el 18 de octubre]. 6. Gaze C, Kepley HO, Walkup JT. Co-occurring psychiatric disorders in children and adolescents with Tourette syndrome. J Child Neurol. 2006;21(8):657-64. 7. Franklin ME, Best SH, Wilson MA, Loew B, Compton SN. Habit reversal training and acceptance and commitment therapy for Tourette syndrome: A pilot project. J Dev Phys Disabil. 2011; 23(1):49-60.

8. Gagné J-P. The psychology of Tourette disorder: Revisiting the past and moving toward a cognitively-oriented future. Clin Psychol Rev. 2018. pii: S02727358(18)30245-9. doi: 10.1016/j.cpr.2018.09.005.

9. Cutler D, Murphy T, Gilmour J, Heyman I. The quality of life of young people with Tourette syndrome. Child Care Health Dev. 2009;35(4):496-504.

10. Lee MY, Mu PF, Wang WS, Wang HS. 'Living with tics': self-experience of adolescents with Tourette syndrome during peer interaction. J Clin Nurs. 2016;25(34):463-71.

11. Mead, G.H. Mind self and society from the standpoint of a social behaviorist
Mind. Chicago: University of Chicago Press; 1934. p. 135-209.

12. Charon JM, Cahill S. Symbolic interactionism: An introduction, an interpretation, an integration. Englewood Cliffs, NJ: Prentice-hall; 1979.

13. O'hare D, Eapen V, Grove R, Helmes E, McBain K, Reece J. Youth with Tourette syndrome: parental perceptions and experiences in the Australian context. Aust J Psychol. 2017;69(1):48-57.

14. Stewart SB, Greene DJ, Lessov-Schlaggar CN, Church JA, Schlaggar BL. Clinical correlates of parenting stress in children with Tourette syndrome and in typically developing children. Pediatrics. 2015;166(5):1297-302.

15. Lee MY, Wang HS, Chen CJ, Lee MH. Social adjustment experiences of adolescents with Tourette Syndrome. J Clin Nurs. 2018. doi:10.1111/jocn.14564.
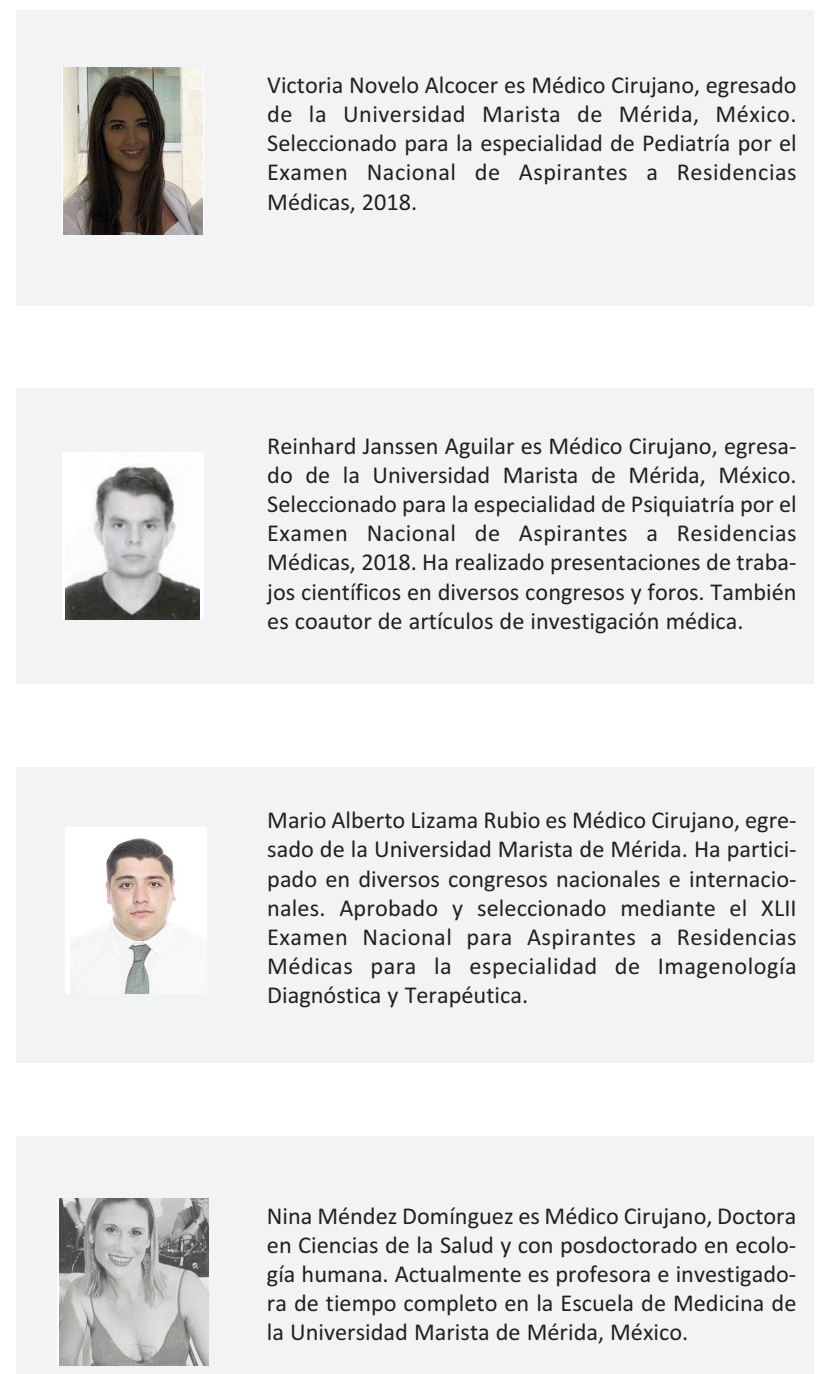

Nina Méndez Domínguez es Médico Cirujano, Doctora en Ciencias de la Salud y con posdoctorado en ecología humana. Actualmente es profesora e investigadora de tiempo completo en la Escuela de Medicina de la Universidad Marista de Mérida, México. 